\title{
Resonancias actuales de la comunidad: el teatro comunitario argentino como espacio de recreación de lazos de pertenencia
} Current resonances of the community: community argentine theater as a space of recreation of ties of belonging

Andrea Romina Sánchez Salinas rominasanchezsalinas@gmail.com https://orcid.org/0000-0001-5269-900X Instituto Multidisciplinario de Estudios Sociales Contemporáneos;

Facultad de Filosofía y Letras; Universidad Nacional de Cuyo/ Consejo Nacional de Investigaciones Científicas y Técnicas (Argentina)

\section{Resumen}

El artículo reflexiona acerca del tipo de comunidad que recrean los grupos de teatro comunitario de Argentina en las primeras dos décadas del siglo XXI. Se analiza el fenómeno como una forma de comunidad tomando formulaciones clásicas de la sociología, especialmente la polaridad conceptual comunidad/sociedad, como así también versiones contemporáneas. El recorrido muestra que los grupos de teatro comunitario constituyen espacios que compensan las lógicas individualistas e impersonales de la racionalidad moderna. Además, por ser un fenómeno que ha alcanzado una presencia y reconocimiento significativos dentro de las políticas culturales públicas, se indaga en la relación de los grupos con el Estado, particularmente en programas y experiencias que proponen instancias de co-gestión que 
desafían la dualidad Estado/sociedad civil. Se problematiza en ese contexto la definición de "comunidad" que se reactiva de forma permanente a lo largo del tiempo tanto en el plano gubernamental como en el organizacional. Desde esta perspectiva, se interpreta a los grupos de teatro comunitario dentro de una red compleja de relaciones sociales entre lo público y lo privado donde se convierten en actores sociales y políticos que asumen funciones de gobierno, como parte de un proceso de desplazamiento desde formas formales hacia formas informales de gobierno.

Palabras clave: comunidad; sociedad; estado; teatro comunitario.

\section{Abstract}

This article reflects on the kind of community recreated by the community theater groups of Argentina during the first two decades of the XXI century. The phenomenon is analyzed as a way of community through some of the classical formulations of Sociology, especially, the conceptual polarity community/society, as well as other contemporary versions of these concepts. This path shows that the community theater groups create spaces that compensate the individualistic and impersonal logic of modern rationality. As this phenomenon has reached a significant presence and acknowledgment among public policies, the present article will analyze the relationship between these groups and the State, especially in those programs and experiences involving co-management, where the duality State/Civil Society is challenged. In this context, this work discusses the definition of "community" which is constantly reviewed at governmental and organizational scale. From such perspective, we interpret the community theater groups as part of a complex network of social relationships between the public and the private spaces, where community theater groups become social and political actors and take some government functions as part of a process of derivation from formal ways to informal ways of government.

Keywords: community; society; state; community theater.

Este trabajo se propone reflexionar sobre la idea de comunidad a propósito de la proliferación de motivos, vocabularios y formas de acción colectiva que se invocan bajo ese nombre tanto desde los gobiernos, Estados y organismos internacionales como desde numerosas 
organizaciones sociales y culturales. Dentro de este conjunto, observamos un fenómeno que ha tenido un crecimiento constante en la Argentina en las primeras décadas del siglo XXI: el teatro comunitario, definido por sus propios actores como teatro de vecinos para vecinos. Los grupos de teatro comunitario están conformados por personas no profesionales del teatro que cuentan la historia de su lugar (pueblo, ciudad o barrio) a través de una representación teatral (Bidegain, 2007). Nos interesa particularmente este fenómeno porque son colectivos que en sus discursos y en sus prácticas evocan, defienden y promueven el ideal comunitario. ¿Qué hay detrás de ese ideal comunitario? ¿Qué tipo de vínculo social recrean en sus prácticas? ¿Es lo mismo hablar de una comunidad de vecinos y vecinas que se reúnen a hacer teatro en su barrio a hablar de una comunidad virtual de amantes del animé? ¿Cómo podemos interpretar este fenómeno que se denomina comunitario en la actual configuración de lo social?

Para aproximar una respuesta a estos interrogantes nos valdremos de algunas conceptualizaciones clásicas de comunidad (Ferdinand Tönnies, Emile Durkheim) y también de formulaciones más recientes (Gabriela Vargas Cetina, Michel Maffesoli) a fin de interpretar el teatro comunitario como una forma de comunidad. También nos interesa reflexionar en torno a espacios liminales del fenómeno (Victor Turner, lleana Diéguez Caballero), que podríamos entender como intersticios entre políticas culturales e iniciativas comunitarias, en el marco de la complejización del entramado de relaciones sociales entre lo público y lo privado (de Marinis, 2005). El objetivo es aportar a las discusiones sobre las múltiples y permanentes evocaciones de comunidad que parecen reactivarse continua e inevitablemente a lo largo del tiempo.

\section{El teatro comunitario}

Debido a la extensión que ha alcanzado el fenómeno del teatro comunitario a nivel nacional e internacional (1) encontramos una vasta producción académica que lo ha estudiado desde distintas disciplinas. Dentro del área de estudios teatrales ha sido abordado como objeto de investigación por diversos autores (Proaño Gómez, 2006, 2013; Bidegain, 2007, 2014, 2017; Rosemberg, 2009; Scher, 2010), que han observado en esta experiencia un proceso cultural transformador en cuanto a la inserción de la práctica en la vida cotidiana de la comunidad en la que trabaja. Bidegain realiza una reconstrucción histórica y una aproximación teatral al fenómeno y Proaño Gómez lo aborda desde la filosofía estética. Otros autores lo analizan desde una perspectiva sociológica y lo interpretan como movimiento (Greco, 2008; Fernández, 2013, 2014, 2017; Elgoyhen, 2014, Falzari, 2014; Sánchez Salinas, 2014) al inscribirlo dentro 
de la multiplicidad de luchas sociales, de movimientos territoriales y acciones sindicales que han proliferado en las últimas décadas a nivel regional y nacional (Svampa, 2009). Retomando estos distintos abordajes, realizamos una breve presentación del contexto de surgimiento y de sus características para presentar luego algunas interpretaciones del fenómeno siguiendo los objetivos expuestos.

\section{Contexto de surgimiento}

La definición de teatro comunitario como teatro de vecinos para vecinos o de la comunidad para la comunidad se vincula en Argentina a la aparición del grupo Catalinas Sur (1983) y a la experiencia posterior del Circuito Cultural Barracas (1996), ambos de la Ciudad Autónoma de Buenos Aires (2). En julio de 1983 comienza la historia del grupo Catalinas Sur, que era el nombre de un complejo de edificios del barrio porteño de La Boca. Allí un grupo de vecinos, miembros de la cooperadora de una escuela, le propusieron a Adhemar Bianchi que dictara un taller de teatro (3) (Bidegain, 2007, 2008; Scher, 2010). Bianchi, que venía hacía tiempo cuestionando algunas formas de la docencia teatral y del teatro independiente, no estuvo de acuerdo con la idea de dar un taller y propuso, en cambio, hacer teatro en la plaza del barrio. Tanto la propuesta de actuar de los miembros de la cooperadora como el giro que produce Bianchi en su carrera teatral, con la consecuente creación de un grupo de vecinos y vecinas que deciden hacer teatro en una plaza, se relaciona con el retorno de la democracia: refleja la necesidad de volver a participar, de decir basta al miedo, al encierro y al silencio impuestos por las dictaduras cívico-militares (4) en la sociedad argentina. No es posible pensar el inicio del teatro comunitario separado de este contexto nacional donde empezaban a consolidarse las movilizaciones por los derechos humanos, y los partidos políticos recuperaban su voz y volvían a la escena pública. A su vez, a escala regional los movimientos sociales se renovaban al tomar distancia de los "viejos movimientos" y ponían en el centro de la escena el concepto de "comunidad", abogando por una transformación social más referida a un cambio de valores que a una lucha política por la conquista del poder del Estado. En este contexto, se ha inscripto el teatro comunitario como un nuevo movimiento sociocultural en tanto propuesta artística que trasciende un objetivo estético.

En 2002 surgieron diez nuevos grupos en el seno de la crisis que atravesaba el país. Algunos nacieron a partir del proyecto de la Carpa Cultural Itinerante del Gobierno de la Ciudad de Buenos Aires (GCBA) que tenía como objetivo entusiasmar a gente de otros barrios para que 
se desarrollen nuevos grupos, otros se desprendieron de asambleas barriales y por la necesidad de reunirse y ocuparse de las distintas problemáticas públicas. En esta década el fenómeno se propagó por varias ciudades de la provincia de Buenos Aires y alcanzó nuevas provincias: Misiones (5), Mendoza, Córdoba, Catamarca. De este modo el escenario político regional luego de los noventa fue propicio para la consolidación de lo que se ha denominado "segunda generación de grupos de teatro comunitario" (Bidegain, 2014: 11) marcado por los discursos críticos al neoliberalismo, la circulación de prácticas contestatarias ligadas a la acción directa y la emergencia de gobiernos de centroizquierda. Desde entonces, el nuevo clima de participación social, de multiplicación de partidos políticos, de recuperación de espacios de debate y de acción, estimuló y favoreció el crecimiento exponencial del teatro comunitario en Argentina, al punto de alcanzar en 2010 casi cincuenta grupos de estas características. Desde ese año el número de grupos se mantiene pero con un alto recambio: grupos nuevos aparecen, otros resurgen y otros se disuelven.

Es posible que la emergencia de políticas públicas de fomento a estas experiencias artísticas comunitarias en los últimos años, así como la creación de instancias de gestión participativa en la implementación de programas y legislaciones del sector, explique las trayectorias de muchos grupos. Retomaremos estas argumentaciones en torno a lo público y al Estado en la segunda parte del trabajo, luego de presentar algunas interpretaciones de lo expuesto a la luz de los pensadores clásicos de la sociología.

\section{El cristal de los clásicos}

Una primera aproximación a la relación del tipo de vínculo social que recrea el teatro comunitario con los esquemas interpretativos clásicos de la sociología fue apuntada por Elgoyhen (2014) quien señala que "al rehabilitar lo comunitario como alternativa a la sociedad moderna actual, el teatro comunitario remite a la oposición clásica entre la 'comunidad' y la 'sociedad' conceptualizada por Ferdinand Tönnies, Max Weber y Émile Durkheim" (2014: 48) (6). La autora sostiene que la edificación de las comunidades de vecinos-actores es regulada por la búsqueda de una sociedad ideal basada en la solidaridad, la cooperación y la primacía de lo colectivo. Así incluye al teatro comunitario dentro de un conjunto de experimentaciones que revalorizan la comunidad como modalidad de organización y producción, e ideal regulatorio de las relaciones humanas. Compartimos la idea de que en estos grupos de teatro se rehabilitan los vínculos comunitarios y que la base de esa construcción está motivada por 
plantear una alternativa a la organización de la sociedad actual. Ahora bien, entendemos que este tipo de grupos no recrean comunidades en su sentido clásico sino más bien representan una instancia colectiva que convive y en cierto modo, facilita las formas societales modernas. Podrían interpretarse como espacios donde los vínculos comunitarios funcionan como posibles paliativos a la inminente desintegración y enfriamiento de las relaciones que trae aparejada la sociedad moderna, como pensaban los autores de la sociología clásica.

Ferdinand Tönnies (1855-1936), en su orientación al movimiento cooperativo, llamaba communities a esas formas de unión social en las que los sujetos articulaban por la vía de la participación democrática, valores y metas hacia los que se sentían vinculados colectiva e igualitariamente. Estas comunidades, corporaciones o sindicatos, al tiempo que evitaban la disolución de relaciones sociales comunitarias se adecuaban a las condiciones de la era industrial (Honneth, 1999). La concepción de Töonies es más próxima a la idea de Community norteamericana que a la de Gemeinschaft alemana. En la tradición anglosajona las communities forman parte del proceso de recomunización y fomentan la acción participativa de los ciudadanos; la sociedad moderna no es un lugar hostil a las comunidades si no el lugar que las alberga. En esta tradición un sistema social que funciona es aquel en el que el individuo participa, por tanto rechazan cualquier imposición externa de organizaciones o instituciones intermedias.

Thomas (1918), consideraba que era un error suponer que los centros establecidos por agencias estatales o de la sociedad civil podían cumplir funciones sociales comunitarias al modo en que, por ejemplo, lo había hecho la parroquia polaca en el viejo mundo. Estas iniciativas resultaban instituciones impuestas por el exterior en lugar de haberse desarrollado libremente por la iniciativa y cooperación de los involucrados: "Quienes estaban al frente de ellas solían saber poco de las tradiciones, de las actitudes y de la lengua nativa de la gente con la que trataban y, por lo tanto, no podían convertirse en líderes sociales genuinos" (Grondona, 2012: 196). Estas afirmaciones nos permiten entender algunas especificidades de las dinámicas de los grupos de teatro comunitario, especialmente las relativas al trabajo en territorio, con problemáticas que afectan directamente a sus participantes y coordinados por un director local que en la mayoría de los casos pertenece al mismo ámbito. Muchos de los grupos de teatro comunitario que han sido coordinados por referentes que no habitan el territorio donde conforman los grupos, han desaparecido (Sánchez Salinas, 2018). Esta idea habilita una posible interpretación del fenómeno con la idea de community en la tradición anglosajona, en el sentido de que son grupos que no funcionan como una imposición externa al territorio. 
Encontramos algunas nociones próximas en Durkheim (1858-1917) quien, también preocupado por los problemas de integración en la sociedad industrial, advirtió la necesidad de implementar unos mecanismos correctivos. Tales propuestas tenían como fin impedir el peligro de desorganización moral producto de la división social del trabajo. En Lecciones de Sociología (1912) propone la creación de unos grupos intermediarios que permitan institucionalizar los conflictos y que al mismo tiempo funcionen como reguladores de las relaciones entre los individuos y el Estado. Esta propuesta surge a partir de diagnosticar que "nuestra enfermedad política depende de la misma causa que nuestra enfermedad social: la ausencia de cuadros secundarios intercalados entre el individuo y el Estado" (Durkheim, 2003: 159). La función de estos grupos sería doble: garantizar tanto la autonomía del Estado como los procesos de individualización moral (el Estado debe liberar las personalidades individuales de los particularismos colectivos). Estos espacios denominados "grupos sociales secundarios" (Durkheim, 2003: 108), profesionales o de base territorial, nos remiten a una de las aristas del fenómeno que nos ocupa: su organización en red. Los grupos de teatro comunitario articulan sus experiencias conformando redes a nivel local y nacional a fin de fortalecer sus procesos territoriales, compartir problemáticas y otorgarle visibilidad al fenómeno. La modalidad en red enlaza a los grupos y conforma actores colectivos capaces de disputar recursos y derechos con el Estado, cumpliendo una función similar a la que Durkheim imaginaba para los grupos intermediarios. Veamos algunos ejemplos que nos permitan clarificar esta interpretación.

En el año 2009 la Red Nacional de Teatro Comunitario logró implementar el Concurso Nacional de Teatro Comunitario en el Instituto Nacional del Teatro (7), con la particularidad de contar con un jurado especial para la evaluación de las propuestas de arte comunitario, bien diferenciadas de las de "teatro off" o "teatro independiente". El concurso vehiculizaba fondos a los ganadores para montaje de espectáculos, sostenimiento de la actividad, compra de equipamiento técnico, capacitación, etcétera. Otro logro de la Red fue la Ley de Promoción al Teatro Comunitario, aprobada en diciembre de 2014 por la Legislatura de la Ciudad de Buenos Aires, que otorga apoyo económico anual a los grupos de la Ciudad Autónoma de Buenos Aires (CABA) a través de PROTEATRO (8). A nivel internacional, la Red articula con otras experiencias que promueven la transformación social desde la práctica artística y comunitaria, aunadas desde 2003 en la Red Latinoamericana de Arte para la Transformación Social y en el Movimiento de Cultura Viva Comunitaria (9).

Otra dimensión de la teoría durkheimiana que ilumina una característica muy particular del tipo de comunidad que estudiamos, y que la diferencia principalmente de otras, es la teatralidad. El carácter ritual del teatro y el proceso de creación de una obra colectiva que reconstruye la 
historia propia, cumple una función de integración simbólica y construcción de identidad. El símbolo, afirma Durkheim (1992), posee la facultad de congregar y recrear un sentimiento de comunidad que consolida una identidad social. En la recreación de rituales colectivos, los individuos reviven los ideales comunitarios. Estos espacios pueden entenderse como instancias de efervescencia colectiva, como dispositivos de tonalidad comunitaria donde se entibian los lazos sociales refrigerados por la sociedad moderna.

Dijimos que esta tendencia a la fusión colectiva o las instancias que recrean los vínculos comunitarios son vistas en los autores clásicos como posibles paliativos a la inminente desintegración y enfriamiento de las relaciones que trae aparejada la modernidad. Encontramos un recelo semejante a esos vínculos societales neoliberales en los colectivos de teatro comunitario, sumado al carácter utópico de sus ideales y al tinte nostálgico de un tiempo pasado. El lazo que no se corta entre aquella época y la actual es la centralidad en "lo comunitario", con una valoración positiva y un potencial de resolución de problemas de amplia proyección. Raymond Williams (2009) observó que lo notable de la comunidad es que es algo que "siempre ha sido"; y Zygmunt Bauman añade "o que siempre existirá en el futuro" (2005: 9). Veamos a continuación qué aportes encontramos en pensadores contemporáneos que reversionan el concepto de comunidad.

\section{De tribus y alegrías}

Michel Maffesoli, pensador posmoderno con una fuerte herencia durkheimiana, utiliza la metáfora del tribalismo como marco teórico para elucidar la lógica específica de esta variada gama de fenómenos sociales emergentes de las sociedades contemporáneas. "El tribalismo nos recuerda, empíricamente, la importancia del sentimiento de pertenencia, a un lugar, a un grupo, como fundamento esencial de toda vida social" (Maffesoli, 2004: 20). Una clave o "carácter esencial" que le atribuye al neotribalismo posmoderno es precisamente la dimensión comunitaria de la socialidad: contrariamente a lo social racionalmente pensado y organizado, la socialidad no es más que una concentración de pequeñas tribus. Así, por medio del "paradigma tribal" se designarían unos modos de relación social de tipo empático que testimoniarían las crisis de un proyecto social en tensión de futuro y la vitalidad de un sentimiento de comunidad ligado a lo presente (Carretero Pasín, 2003: 110). Para Maffesoli, el tribalismo marcará la dinámica de las sociedades en los próximos decenios. Bajo esta perspectiva ya no es a partir del individuo que se constituye la vida en sociedad si no que ésta es, ante todo, "emocional, 
fusional, gregaria" (Maffesoli, 2004: 22). Hay en él un intento de rememorar que el ser humano es comunitario:

El poner durante mucho tiempo el acento en el individuo, y después en el individuo social, ha hecho olvidar lo que, precisamente, es primordial para el hombre: el vivir en común. Más allá de lo social, donde se expresa la solidaridad mecánica, encontramos la socialidad, que remite a la solidaridad orgánica, donde la relación con el cosmos y la relación con el otro se entretejen sin cesar (Maffesoli, 1996: 15).

La fuerza de los mitos, de las religiones, de las utopías, radicaría más en su forma que en su contenido, en su capacidad para interpenetrar la conciencia colectiva y garantizar una socialidad. En esta perspectiva, lo imaginario es contemplado como un continente de acogida que proporciona una identidad social, como un espacio que conforma una congregación comunitaria en torno a emblemas simbólicos (Carretero Pasín, 2003).

Tanto el optimismo y vitalismo de las ideas de Maffesoli como su concepción de "lo imaginario" nos remite a la discursividad de la utopía teatral (10) comunitaria. Cuando el autor apunta a que lo imaginario es aquello que dota de vigor a la utopía al movilizar la potencia social y cuestionar de este modo el orden establecido, nos recuerda la convicción de los teatreros comunitarios acerca de que la creación de una obra de arte implica la invención de un universo con reglas propias que remite al mundo real, pero que no lo copia tal cual es, sino que traduce un punto de vista (Scher, 2010). Esta operación implica para ellos poner en marcha la creatividad para pensar la opinión que van a dar de esa "realidad" que se nos presenta como natural e inmutable. De esta manera el creador tiene la posibilidad de "pensar el mundo fuera de los límites de lo impuesto y allí empieza la transformación" (Scher, 2010: 87). Proaño Gómez observa que en el teatro comunitario encontramos una reversión del concepto de mímesis: "lo que se reproduce no es una mera copia de lo que se critica, sino que tales materiales están transformados para mostrar aspectos ocultos de la realidad o de la historia política" (2013: 259). Esto es posible, explica la autora, por los recursos empleados en las obras de teatro comunitario que distorsionan la mímesis realista (la reconstrucción histórica a partir de lo que recuerdan los integrantes de los grupos, la estructura no lineal de las obras, el uso de la metáfora, la sátira, el grotesco, entre otras) (11).

Estas reflexiones nos invitan a interpretar a los grupos de teatro comunitario como neotribus contemporáneas: despiertan la dimensión comunitaria de la socialidad, inducen otra relación respecto a la alteridad; incluso a través del juego teatral se "pone en escena el origen, la 
fuente, lo primitivo y lo bárbaro (...) al redinamizar, de manera no siempre consciente, un cuerpo social un tanto envejecido" (Maffesoli, 2004: 18). Si bien podemos reconocer el énfasis actual en la dimensión comunitaria de la sociabilidad, nos preguntamos junto a otros ¿La comunidad no ha sido también "reactivada y reinventada por las racionalidades políticas contemporáneas"? (de Marinis, 2005: 1) ¿Cómo interpretar el uso extendido y positivizado de lo comunitario por parte del Estado? Estas preguntas nos invitan ir más allá de una aparente masa de neotribus fusionales y gregarias.

\section{Ser o no ser: los espacios liminales}

En aras de problematizar el uso extendido y generalizado del concepto de comunidad, Vargas Cetina (2004) propone el concepto de "interfaz" para referirse a cierto tipo de comunidades no fundadas en la identidad de sus miembros sino más bien, en la diferencia. Considera que el concepto clásico de comunidad no es válido para pensar algunas asociaciones actuales ya que contiene resonancias utópicas que difícilmente sean aplicables a las nuevas formas de organización (ONG, redes de comunicación por internet, entre otras). Estas nuevas formas de asociación son efímeras, de membresía voluntaria, de objetivos y composición cambiantes y de jerarquía relativamente débil. Por tanto, para Vargas Cetina, el concepto de comunidad no puede aplicarse a estas agrupaciones humanas, puesto que se basa en imaginarios que evocan ideas de igualdad (o al menos mismidad), en un territorio común y en un ideal de armonía que poco tiene que ver con las formas actuales de asociación (Vargas Cetina, 2004).

Muy por el contrario, observamos que tales características son justamente las que buscan los colectivos de teatro comunitario: sus miembros evocan, defienden y promueven los valores de igualdad y solidaridad, apelan al ejercicio de la memoria colectiva para reconstruir la historia local, y, por último, se identifican y asientan en un territorio determinado (generalmente, el barrio) que intervienen con presentaciones artísticas en el espacio público (12). Además, el componente utópico está presente en todas sus prácticas y formulaciones, siempre ligado al poder transformador del arte. Muchos de ellos están convencidos de que el arte no es una herramienta de transformación social, sino que es transformador en sí mismo. Como afirma la directora de Matemurga de Villa Crespo:

La dignificación de la condición humana de la comunidad, su carácter activo, el conocimiento del otro, los lazos solidarios que se generan a partir de la necesidad de poner 
cada uno su parte, el surgimiento de una sociedad creativa, no son cuestiones que estén por fuera de la tarea artística, sino que le son inherentes (...).

Cuando una comunidad desarrolla su creatividad, con toda la amplitud que este concepto implica, comienza a dejar de ser espectadora de su destino para pasar a ser parte activa de la vida social. El arte es parte central de esta transformación (Scher, 2010: 68 y 88).

Ahora bien, el concepto de "interfaz" quizás sí nos ayude a comprender algunos espacios liminales del teatro comunitario, entendidos como instancias de participación conjunta entre organizaciones y programas estatales que son promovidas tanto por colectivos de organizaciones culturales (Movimiento de Cultura Viva Comunitaria) como por el Estado (Consejo Cultural Comunitario del Programa Puntos de Cultura) (13). Usamos el concepto de liminalidad en tanto los consideramos espacios que no pueden definirse de forma acabada ni vincularse a un territorio único, ni tampoco poseen una naturaleza estatal u organizacional exclusivamente.

El concepto de lo liminal en antropología es utilizado para estudiar las características sociales del ritual, analizando la liminalidad en esas situaciones ambiguas o pasajeras, de límite o frontera entre dos campos. Se le adjudican algunas condiciones como la realización de una experiencia en los intersticios de dos mundos y la creación de communitas, entendida como una antiestructura en la que se suspenden las jerarquías, a la manera de "sociedades abiertas" donde se establecen relaciones igualitarias espontáneas y no racionales (Diéguez Caballero, 2007: 38). Nos valemos de este concepto para pensar los espacios donde se combinan, por un lado, la promoción de formas de participación comunitaria fomentadas por los gobiernos en tanto elemento vital de la democracia; y por el otro, el incentivo a esa participación activa desde los grupos y colectivos que buscan la recreación de vínculos comunitarios y la constitución de sujetos comprometidos con su realidad. De ello resultan unos "entes liminales" (Diéguez Caballero, 2007: 38) donde se diseñan estrategias de intervención en la esfera pública de conveniencia mutua. Concretamente, esos espacios son los consejos, comisiones, encuentros, asambleas, foros y actividades donde se ponen en práctica tales estrategias.

El Movimiento de Cultura Viva Comunitaria, por ejemplo, representa una multitud muy numerosa de agrupaciones que al tiempo que sostienen un trabajo territorial, se vinculan a una red internacional que demanda a los gobiernos locales la aprobación de un presupuesto fijo destinado a la "cultura comunitaria". En el seno de esa red se libró la discusión en los años 2014 y 2015 por la Ley Nacional de las Culturas en Argentina, que provocó distintas tensiones entre las organizaciones y los agentes de gobierno. A su vez, esa ley fue un proyecto que nació 
del Frente de Artistas y Trabajadores de la Cultura, compuesto por artistas independientes y por otros "institucionalizados" (empleados de organismos públicos de cultura, de centros culturales oficiales, entre otros). Muchos grupos de teatro comunitario participaron de ambos espacios, a veces representados por la Red Nacional y otros de forma individual.

El Consejo Cultural Comunitario creado por el Programa Puntos de Cultura en 2016 es una plataforma de acción sostenida por el Estado en la que participan representantes de organizaciones, redes y colectivos culturales. Tiene como misión la articulación con la Dirección Nacional de Diversidad y Cultura Comunitaria del Ministerio de Cultura de la Nación y su principal objetivo es diseñar -a través de instancias abiertas y participativas en todo el país- el Plan Nacional de Cultura Comunitaria 2018-2028. Dicho plan, elaborado con participación de todas las redes y organizaciones que trabajan a nivel nacional y regional, presentará líneas de trabajo y propuestas pensadas específicamente para el fortalecimiento y desarrollo del sector de la cultura comunitaria, instrumentos formativos, de financiación, promoción y articulación con el Estado. El Consejo, además de estar constituido por representantes de todas las regiones a través de la Comisión Nacional de Puntos de Cultura, tiene representación de distintas redes temáticas (14) dentro de las cuales se encuentra la Red Nacional de Teatro Comunitario. La Comisión Nacional de Puntos de Cultura, también constituye uno de estos espacios donde Estado y organizaciones discuten las políticas del sector, pero a diferencia del Consejo, la Comisión trabaja con representantes de nodos regionales (15) con el objetivo de acompañar el trabajo y el fortalecimiento de los Puntos de Cultura, a través de instancias de co-gestión y participación definidas con el Estado.

Como vemos, estos espacios admiten múltiples interpretaciones, también podrían pensarse como "grupos intermediarios", como actores corporativos, como políticas socioculturales participativas, o bien como public-private partnerships (asociaciones público-privadas), donde "las comunidades son convocadas (o ellas mismas se convocan) a tareas de gobierno" (de Marinis, 2005: 26). Si de un esquema se tratase, podríamos dibujar la ubicación de estos actores en relación a su cercanía o distanciamiento con el Estado. Pero de alguna manera seguiríamos atravesados por la mirada que establece un parteaguas entre Estado/sociedad civil/mercado y justamente lo que nos interesa aquí es interpretarlo en otra clave.

Creemos que la proliferación de estos espacios está posibilitada por un cambio en la articulación de actores sociales y políticos, muy vinculado a la declinación del Estado de Bienestar. Como lo formulan Lemke, Krasmann y Bröckling: 
Lo que se puede observar no es una disminución de la soberanía estatal o de sus capacidades de planificación, sino un desplazamiento desde formas formales hacia formas informales de gobierno. Esto comprende la "relocalización de modelos de acción definidos estatalmente hacia niveles supraestatales o de nuevas formas de 'subpolitica', que operan por 'debajo' de aquello que tradicionalmente constituyó lo político (de Marinis, 2005: 20).

Preferimos adoptar esta mirada que se ubica en el marco de la complejización del entramado de relaciones sociales entre lo público y lo privado, donde se recodifica el rol del Estado al tiempo que erigen nuevos espacios sociales de intervención y nuevos actores que desafían la lógica dual de "Estado" y "Sociedad Civil". Así, entendemos al teatro comunitario y sus extensiones como parte de esa densa red que contribuye a cierta "economización" que el Estado realiza de sus propios medios de gobierno, esto es, como parte de esos nuevos actores sociales y políticos que asumen funciones de gobierno. Esta mirada no pretende ignorar su potencial como espacio de resistencia, de reconstrucción de la memoria colectiva, de construcción de sentido e identidad; sino más bien, procura estar atentos a distinguir tales elementos emancipatorios de nuevas modalidades de dominación.

\section{Conclusiones}

El recorrido ha procurado dotarnos de herramientas conceptuales y modos de inteligibilidad para comprender mejor las comunidades en sus configuraciones actuales, específicamente las comunidades que recrean los grupos de teatro comunitario en la Argentina. Las conceptualizaciones clásicas de comunidad nos han valido para comprender que a pesar de una aparente reactivación actual del concepto por parte de organizaciones e instituciones de gobierno, la comunidad siempre ha sido y seguirá siendo convocada como un modelo ideal de organización social. Asimismo, resulta un dispositivo que funciona como un espacio que entibia las relaciones impersonales y las motivaciones competitivas de la racionalidad moderna. En este sentido, los grupos de teatro comunitario no recrean comunidades en su sentido clásico sino más bien representan una instancia colectiva que convive y en cierto modo, facilita las formas societales modernas.

Las conceptualizaciones también nos han servido para visualizar el rol que cumplen en la sociedad moderna estos espacios comunitarios o grupos intermediarios en relación al Estado. Para responder a ello hemos apelado a una mirada más general y menos binaria, que nos 
permite visualizar espacios liminales donde se disputan tareas de gobierno en un proceso "economización" que opera el Estado. Así, las propuestas de co-gestión de programas públicos y organizaciones se interpretan como un desplazamiento de medios formales a informales de gobierno, donde los grupos de teatro comunitario se convierten en actores sociales y políticos que asumen tareas de gobierno en sus territorios. Para llegar a esta conclusión fue necesario apartarnos de aquellas miradas duales que entienden al fenómeno como un espacio de resistencia únicamente y que a la larga invisibilizan los intersticios donde se diseñan, discuten y disputan los modos en que se realizan aquellas tareas.

Ante grandes preguntas como la de si el teatro comunitario ha sido una resistencia "a la penetración en la sociedad argentina de la lógica individualista y materialista del capitalismo" (Elgoyhen, 2014: 49) o si es un engranaje más en la construcción de una sociedad democrática y participativa, podríamos decir que el teatro comunitario constituye una apuesta, un gesto, un avance (16) hacia esa comunidad del entendimiento común y la armonía. $Y$ en esa búsqueda observamos que aparecen modalidades disímiles a lógicas individualistas o verticalistas donde prima el nebeneinander, donde permanecemos separados a pesar de todas las uniones (17). El hecho de que ese intento pueda ser interpretado como una nueva forma de dominación del capitalismo tardío "a través de la instrumentalización de lealtades personales y de activas responsabilidades" (de Marinis, 2005); o como una batalla cultural al neoliberalismo con notables elementos emancipatorios, en última instancia, depende de un posicionamiento político-ideológico. Partiendo del supuesto de que si lo que opera bajo la racionalidad política neoliberal es una reconfiguración de las formas de dominación, cuestionar el orden social vigente interviniendo en su construcción simbólica nos revela un acto singular de construcción de sentido e identidad. Por este motivo, creemos conveniente indagar a futuro en la especificidad de los grupos de teatro comunitario respecto a otras comunidades: su dimensión artística.

\section{Notas}

(1) Entre 2010 y 2017 el número de grupos de teatro comunitario en Argentina ha oscilado entre 45 y 55 grupos, incluyendo en este conjunto a los grupos que integran la Red Nacional de Teatro Comunitario y que deciden asumir esa denominación, o que incluso han surgido a partir del estímulo de otro grupo de la Red (www.teatrocomunitario.com.ar).

(2) La aclaración de los primeros usos de la denominación "teatro comunitario" es necesaria en tanto existen algunas controversias en torno a cuál fue el primer grupo de teatro de vecino/as o de teatro comunitario (y dónde). Por 
cuestiones de extensión, nos limitaremos a aclarar aquí que entendemos que las experiencias de teatro anarquista, teatro popular, teatro participativo, teatro callejero, teatro del oprimido y teatro independiente, fueron condición de posibilidad del nacimiento del teatro comunitario actual. Para ampliar en estos antecedentes ver (Bidegain, 2007; Scher, 2010; Fos, 2011; Fernández, 2013; Verzero, 2014; Berman, Durán y Jaroslavsky, 2014).

(3) La cooperadora había sido expulsada porque dentro de la escuela no podía funcionar una asociación democrática y autogestiva. La prohibición de asociaciones autónomas dentro de escuelas públicas fue una medida implementada en la gestión del brigadier Osvaldo Cacciatiore, intendente de la última dictadura militar en la Argentina (1976-1983).

(4) La historia de la Argentina del siglo XX, al igual que la de numerosos países de Latinoamérica, está signada por una sucesión de intervenciones militares a gobiernos constitucionales que mantuvieron en el poder a sectores militares con el apoyo de sectores civiles (1930-1932, 1943-1946, 1955-1958, 1962-1963, 1966-1973, 1976-1983). Este proceso de experiencias autoritarias culminó en la dictadura más cruenta del Cono Sur de América Latina en materia de violaciones a los derechos humanos (Catoggio, 2010) con más de 30.000 personas desaparecidas.

(5) Exceptuando la Murga de la Estación de Posadas (1999) y la Murga del Monte de Oberá (2000) que surgen antes de la crisis de 2001.

(6) De modo esquemático podríamos resumir que la polaridad conceptual de "comunidad" y "sociedad" en la sociología clásica se basó en la distinción por el tipo de vínculo de sus miembros (relaciones de parentesco versus relaciones impersonales), por el sentimiento subjetivo de pertenencia (pertenencia al todo versus motivaciones racionales) y por la esencia de la vida en común como algo natural en la comunidad o como algo artificial, ficcional en la sociedad.

(7) El Instituto Nacional del Teatro es el organismo dependiente del Ministerio de Cultura que rige la promoción y apoyo de la actividad teatral en la Argentina, creado a partir de la sanción de la Ley Nacional del Teatro Nro. 24800 en 1997. http://inteatro.gob.ar.

(8) La ley de protección, promoción y difusión del Teatro Comunitario en el ámbito de la Ciudad Autónoma de Buenos Aires le permite a los grupos de teatro comunitario acceder a subsidios de PROTEATRO (Instituto para la Protección y Fomento de la Actividad Teatral No Oficial de la Ciudad de Buenos Aires) dependiente del GCBA. www.buenosaires.gob.ar/proteatro/

(9) El Movimiento de Cultura Viva Comunitaria expresa los acuerdos e iniciativas de más de 1500 organizaciones de Latinoamérica que apuntan al fortalecimiento de los procesos de Cultura Viva Comunitaria. Su estrategia principal es impulsar una campaña continental que apunte al $1 \%$ de presupuestos nacionales para cultura y el $0,1 \%$ para cultura comunitaria. Forman parte de ella colectivos comunitarios, redes sociales y también del campo de la gestión gubernamental y la actividad académica de 17 países de América Latina. http://culturavivacomunitaria.org

(10) Nombre del primer largometraje en formato documental sobre teatro comunitario dirigido por Adolfo Cabanchik que tuvo amplia difusión en el medio y sigue siendo una herramienta de divulgación extendida del fenómeno (Cabanchik, 2006).

(11) Proaño Gómez desarrolla un extendido análisis de los recursos que distorsionan la mímesis realista en las obras de teatro comunitario en su trabajo sobre la estética del fenómeno (Proaño Gómez, 2013: 257-277).

(12) El resaltado intenta aclarar que nos referimos a objetivos y aspiraciones que atraviesan el teatro comunitario como horizonte, no como identidad lograda o características efectivamente reconocibles en los grupos. Por el contrario, algunos estudios han sabido demostrar las infinitas dificultades y contradicciones que aparecen en esa búsqueda de construcción horizontal. Ver Fernández (2013).

(13) El Programa Puntos de Cultura, dependiente de la Dirección Nacional de Diversidad y Cultura Comunitaria del Ministerio de Cultura de la Nación de Argentina, fue implementado en 2011 y toma como referencia la experiencia homónima de Brasil. Apunta a fortalecer organizaciones de la sociedad civil -con personería jurídica o sin ella- que 
trabajan en territorio, con diferentes poblaciones, y que transforman la vida de sus comunidades a través del arte y la cultura.

(14) Las distintas redes y temáticas con representación en el Consejo son: "Colectivos de comunicación popular", "Colectivos artísticos/grupos de teatro comunitario/expresiones del carnaval", "Centros culturales independientes y autogestivos", "Bibliotecas populares, clubes sociales y deportivos y espacios educativos" y "Colectivos de diversidad".

(15) Las regiones culturales de Argentina que forman parte de la Comisión Nacional de Puntos de Cultura son: Noroeste, Noreste, Cuyo, Patagonia, Centro Litoral y Centro.

(16) Es una expresión nativa: "el teatro comunitario avanza sobre las utopías" sostiene Adhemar Bianchi, director de Catalinas Sur en el largometraje La utopía teatral (Cabanchik, 2006).

(17) Töonies emplea esta palabra al describir que "la teoría de la sociedad construye un círculo de hombres que, como en la comunidad, conviven pacíficamente, pero no están esencialmente unidos sino esencialmente separados, y mientras en la comunidad permanecen unidos a pesar de todas las separaciones, en la sociedad permanecen separados a pesar de todas las uniones" (de Marinis, 2010).

\section{Bibliografía}

Bauman, Z. (2005). Comunidad. Buenos aires: Siglo XXI.

Berman, M.; Durán, A. y Jaroslavsky, S. (2014). Pasado y presente de un mundo posible. Del teatro independiente al teatro comunitario. Buenos Aires: Leviatán.

Bidegain, M. (2007). Teatro Comunitario. Resistencia y transformación social. Buenos Aires: Atuel.

Bidegain, M. (2014). Introducción. En El movimiento teatral comunitario argentino. Reflexiones acerca de la experiencia en la última década (2001-2011) (pp. 9-15). CABA: Ediciones del CCC.

Cabanchik, A. (Director). (2006). La utopía teatral. [Largometraje documental]. Buenos Aires.

Carretero Pasín, Á. E. (2003). Una aproximación a la sociología de lo imaginario de Michel Maffesoli. Sociológica (53), pp. 101-119.

Catoggio, M. S. (2010). La última dictadura militar argentina (1976-1983): la ingeniería del terrorismo de Estado. Encyclopedia of Mass Violence. Recuperado de https://www.sciencespo.fr/mass-violence-war-massacre-resistance/en/.

de Marinis, P. (2005). 16 comentarios sobre la(s) sociología(s) y la(s) comunidad(es). Papeles del CEIC, 15, Centro de Estudios sobre la Identidad Colectiva, Universidad del País Vasco, España.

de Marinis, P. (2010). Sociología clásica y comunidad: entre la nostalgia y la utopía (un recorrido por algunos textos de Ferdinand Tönnies). En de Marinis, P.; Gatti, G. \& Irazuzta, I. La comunidad como pretexto. En torno al (re)surgimiento de las 
solidaridades comunitarias (pp. 347-382). Barcelona y México DF: Anthropos y Universidad Autónoma Metropolitana-Iztapalapa.

Diéguez Caballero, I. (2007). Escenarios liminales. CABA: Atuel.

Durkheim, E. (1992). Las formas elementales de la vida religiosa. Madrid: Akal.

Durkheim, E. (2003). Lecciones de Sociología. Buenos Aires: Miño y Dávila.

Elgoyhen, L. (2014). Las dinámicas de movilización colectiva en el movimiento teatral comunitario (pp. 19-74). En El movimiento teatral comunitario argentino. CABA: Ediciones del CCC.

Falzari, G. (2014). Un acercamiento al movimiento teatral comunitario (pp. 75-122). En El movimiento teatral comunitario argentino. CABA: Ediciones del CCC.

Fernández, C. I. (2013). Antecedentes e historia del teatro comunitario argentino contemporáneo. Los inicios de un movimiento. AISTHESIS, 54, pp. 147-174.

Fernández, C. I. (2014). Identidades, memorias y espacios en las prácticas del Grupo de Teatro Popular de Sansinena. En El movimiento teatral comunitario argentino. CABA: Ediciones del CCC, pp. 123-165.

Fernández, C. I.; Bidegain, M. y otros (2017). Estrategias colectivas de gestión cultural: el caso del grupo de teatro comunitario de Rivadavia (pp. 138-192). En Modelos de Gestión Teatral. Buenos Aires: INTEATRO.

Fos, C. (2011). Del teatro anarquista al teatro comunitario actual. Cuadernos de Acción Cultural. Buenos Aires: Artes Escénicas.

Greco, Á. (2008). Teatro Comunitario en la Argentina y la Red Americana de Arte para el Cambio Social. Telón de Fondo. Revista de Teoría y Crítica Teatral, 8.

Grondona, A. (2012). La 'comunidad' de Chicago. Cuestión social, cuestión urbana y cambio social: una sociología de lo comunitario. En de Marinis, P. (coord.) Comunidad: estudios de teoría sociológica (pp.189-228). Buenos Aires: Prometeo.

Honneth, A. (1999). Comunidad: esbozo de una historia conceptual. Isegoría-Revista de Filosofía Moral y Política, 20, pp. 5-15.

Maffesoli, M. (1996). De la orgía. Una aproximación sociológica. Barcelona: Ariel.

Maffesoli, M. (2004). El tiempo de las tribus. Bs As: Siglo XXI editores.

Proaño Gómez, L. (2006). Estética social y la aparición de lo político. Teatro comunitario y espacio urbano. Espacios de representación. Madrid: Fundación Autor.

Proaño Gómez, L. (2013). Teatro y estética comunitaria. Bs As: Biblos.

Rosemberg, D. (2009). Teatro Comunitario Argentino. Buenos Aires: Emergentes Editorial. 
Sánchez Salinas, R. (2014). El teatro comunitario en el proceso de transformación de la sociedad: el caso de Res o no Res en el barrio de Mataderos. En El movimiento teatral comunitario argentino (pp. 167-218). CABA: Ediciones del CCC.

Sánchez Salinas, R. (2018). Las organizaciones culturales y su vínculo con el Estado: el caso del teatro comunitario mendocino. En Segura, M. S. y Prato, V. (comp.). Estado, sociedad civil y políticas culturales. Rupturas y continuidades en Argentina entre 2003 y 2017 (en prensa). Buenos Aires: RGC Ediciones.

Scher, E. (2010). Teatro de vecinos de la comunidad para la comunidad. Buenos Aires: INTeatro.

Svampa, M. (2009). Cambio de época. Movimientos sociales y poder político. Buenos Aires: Siglo XXI Editores.

Vargas Cetina, G. (2004). La asociación efímera. Repensando el concepto de comunidad desde la literatura cyberpunk. Cuadernos de Bioética 11 (sección Doctrina). Recuperado de http://www.bioetica.org/cuadernos/doctrina38.htm

Verzero, L. (2014). Teatro militante. Buenos Aires: Biblos.

Williams, R. (2009). Marxismo y literatura. Buenos Aires: Las Cuarenta. 